\title{
A Hybrid Particle Swarm Optimization (PSO)-Simplex Algorithm for Damage Identification of Delaminated Beams
}

\author{
Xiangdong Qian, ${ }^{1}$ Maosen Cao, ${ }^{1,2}$ \\ Zhongqing $\mathrm{Su}^{3}$ and Jiangang $\mathrm{Chen}^{3}$ \\ ${ }^{1}$ Department of Engineering Mechanics, Hohai University, Nanjing 210098, China \\ ${ }^{2}$ Institute of Fluid Flow Machinery, Polish Academy of Sciences, 80-952 Gdansk, Poland \\ ${ }^{3}$ Department of Mechanical Engineering, The Hong Kong Polytechnic University, Hung Hom, Kowloon, \\ Hong Kong Special Administrative Region, Hong Kong
}

Correspondence should be addressed to Maosen Cao, cmszhy@hhu.edu.cn

Received 5 May 2012; Revised 24 July 2012; Accepted 29 July 2012

Academic Editor: Alessandro Marzani

Copyright (C) 2012 Xiangdong Qian et al. This is an open access article distributed under the Creative Commons Attribution License, which permits unrestricted use, distribution, and reproduction in any medium, provided the original work is properly cited.

Delamination is a type of representative damage in composite structures, severely degrading structural integrity and reliability. The identification of delamination is commonly treated as an issue of nondestructive testing. Differing from existing studies, a hybrid optimization algorithm (HOA), combining particle swarm optimization (PSO) with simplex method (SM), is proposed to identify delamination in laminated beams. The objective function of the optimization problem is created using delamination variables (optimization parameters) together with actually measured modal frequencies. The HOA adopts a hierarchical and cooperative regime of global search and local search to optimize the objective function. The PSO performs global search for objective function space to achieve a preliminary solution specifying a local potential space. Initialized by this preliminary solution, the SM executes local search for the local potential space to explore the optimal solution. The HOA is validated by a series of simulated delamination scenarios, and the results show that it can identify delamination in laminated beams with decent accuracy, reliability and efficiency. The method proposed holds promise for establishing online damage detection system beneficial for health monitoring of laminated composite structures.

\section{Introduction}

Composite structures have been extensively used in aerospace, civil, and mechanical fields due to their superior fatigue characteristics, damage tolerance, and stiffness to weight ratio compared to that of metals [1]. In laminated composite structures, delamination is a type 
of representative damage, commonly occurring as crack in matrix materials, separation of adjoining piles, imperfect bonding, and broken fibers [2]. The presence of delamination may severely influence the mechanical properties of composite structures. Therefore, damage detection of local and tiny delamination in the framework of structural health monitoring (SHM) has become a technological and scientific issue of significant importance [3-7].

Traditionally, delamination identification is performed by model-free nondestructive testing (NDT) methods such as acoustic, ultrasonic, radiographs, magnetic, thermal, and eddy current [8-12]. In spite of distinct capabilities of these methods, their limitations for use in SHM are noteworthy and can be described as (1) a priori knowledge about the vicinity of the delamination is required; (2) ready accessibility to the structural portion of interest is necessary; (3) commonly inapplicable for determining the details of delamination, except its occurrence; and (4) the procedure to carry out these methods is usually time consuming and costly. These deficiencies limit the use of these methods to develop an online system of delamination identification for laminated structures.

As an alternative of model-free NDT methods, model-dependent methods of delamination identification have received increasing interest in the SHM community [3, 13-16]. This type of methods uses analytical or numerical model together with experimentally acquired dynamic responses as damage indictor to identify delamination in a composite structure. Damage indictor is used to calibrate the analytical or numerical model that changes itself with varying delamination variables. A practical damage indictor is modal frequency due to its effectiveness in reflecting delamination [17-19] and facilitation in experimental acquisition.

In general, model-dependent delamination identification can be tactically treated as an optimization problem, for which the objective function is created by relating measured modal frequencies to delamination variables [14-16]. An appropriate optimization method is utilized to optimize the objective function, giving the estimate of delamination parameters. Existing optimization methods can be categorized into local- and global-search methods $[20,21]$. The local-search methods, typically Nelder-Mead simplex method (SM) [22], feature great efficiency in local search due to small cost of function evaluation, but they are somewhat susceptible to local minima [23]. The global-search algorithms, particularly particle swarm optimization (PSO) [24-26], have the merit of searching an optimal solution by casting off local minima along with the inadequacy of low efficiency in convergence.

Differing from existing studies, this study addresses a hybrid optimization algorithm (HOA) featuring cooperative PSO and SM for the identification of delamination in laminated composite beams. The HOA adopts a hierarchical regime of global search using the PSO incorporating local search using the SM to explore an optimal solution indicating delamination. The HOA is demonstrated by a series of simulated delamination cases, and the results show that it can identify delamination in composite beams with great accuracy, reliability, and efficiency.

\section{Optimization Problem Describing Delamination}

A particular optimization problem for delamination identification in laminated composite beams is set up based on Euler-Bernoulli beam theory [27] and delamination indicators referring to modal frequencies. The procedure to formulate the optimization problem, stated below, is applicable to any type of delaminated beams considering different boundary conditions. Without loss of generality, the formulation is exemplified by a double-layered clampedclamped laminated beam bearing a delaminated segment, shown in Figure 1. The beam has 


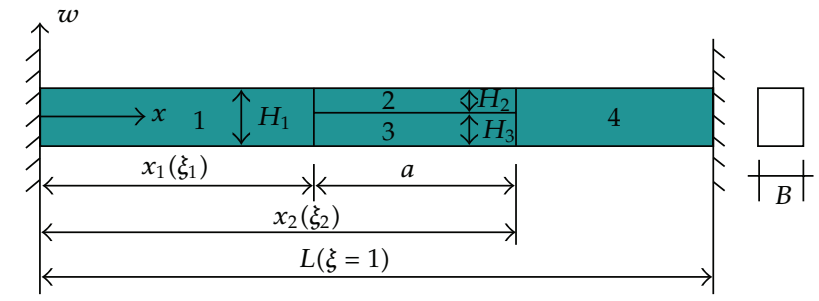

(a)

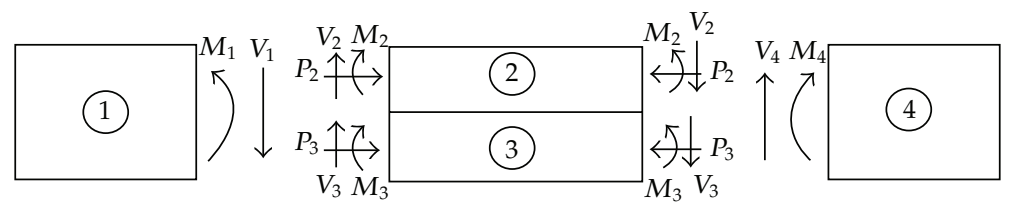

(b)
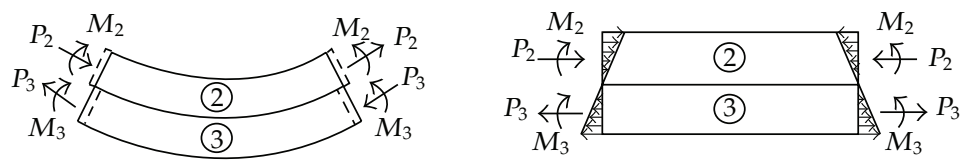

(c)

Figure 1: Model for double-layered clamped-clamped laminated beam bearing a delaminated segment. (a) geometry, (b) free bodies, (c) continuity conditions: compatibility of axial deformations (left) and equilibrium of axial forces (right).

a rectangular cross-section of height $H_{1}$, width $B$, and length $L$, and the delaminated segment of span $a$ consists of Subbeams 2 and 3, specified by elasticity moduli $E_{2}$ and $E_{3}$, density of materials $\rho_{2}$ and $\rho_{3}$, and heights $H_{2}$ and $H_{3}$, respectively. For vibration analysis, the entire beam is treated as a combination of four subbeams, of which each one is modeled as an Euler-Bernoulli beam that is governed by the following equation of motion:

$$
E_{i} I_{i} \frac{\partial^{4} w_{i}(x, t)}{\partial x^{4}}+\rho_{i} A_{i} \frac{\partial^{2} w_{i}(x, t)}{\partial t^{2}}=0, \quad i=1-4
$$

where $w_{i}, E_{i}, \rho_{i}, I_{i}$, and $A_{i}$ denote the transverse displacement, modulus of elasticity, density of material, cross-sectional moment of inertia, and cross-sectional area for the $i$ th subbeam, respectively.

Treating (2.1) using variable separation method, mode shapes of each subbeam can be resulted in the form

$$
\begin{array}{ll}
W_{1}(\xi)=C_{11} \cos \left(\lambda_{1} \xi\right)+C_{12} \sin \left(\lambda_{1} \xi\right)+C_{13} \cosh \left(\lambda_{1} \xi\right)+C_{14} \sinh \left(\lambda_{1} \xi\right), & 0 \leq \xi \leq \xi_{1}, \\
W_{2}(\xi)=C_{21} \cos \left(\lambda_{2} \xi\right)+C_{22} \sin \left(\lambda_{1} \xi\right)+C_{23} \cosh \left(\lambda_{2} \xi\right)+C_{24} \sinh \left(\lambda_{2} \xi\right), & \xi_{1} \leq \xi \leq \xi_{2}, \\
W_{3}(\xi)=C_{31} \cos \left(\lambda_{3} \xi\right)+C_{32} \sin \left(\lambda_{3} \xi\right)+C_{33} \cosh \left(\lambda_{3} \xi\right)+C_{34} \sinh \left(\lambda_{3} \xi\right), & \xi_{1} \leq \xi \leq \xi_{2}, \\
W_{4}(\xi)=C_{41} \cos \left(\lambda_{4} \xi\right)+C_{42} \sin \left(\lambda_{4} \xi\right)+C_{43} \cosh \left(\lambda_{4} \xi\right)+C_{44} \sinh \left(\lambda_{4} \xi\right), & \xi_{2} \leq \xi \leq 1,
\end{array}
$$

where $\xi=x / L$ is the nondimensional $x$-coordinate along beam length, and $\lambda_{i}=L \sqrt[4]{\rho_{i} A_{i} \omega^{2} / E_{i} I_{i}}$ denotes the nondimensional modal frequencies. $C_{2 j}=C_{3 j}$ is considered to specify 
a constrained mode model; that is, subbeams 2 and 3 are stipulated to have identical transverse deformations while they can slide along the delaminated surface. The selection of constrained mode model rather than the free mode model is attributed to the insignificant relative transverse deformation between subbeams 2 and 3 for first several mode shapes, adopted for delamination identification.

An eigenvalue problem can be derived from (2.2) by taking into account the continuity and boundary conditions detailed in Figures 1(b) and 1(c), given as

$$
M\left(E_{2}, E_{3}, \rho_{2}, \rho_{3}, B, H_{2}, H_{3}, \xi_{1}, \xi_{2}, \omega_{i}\right) C=0
$$

where $\xi_{1}$ and $\xi_{2}$ signify the left and right ends of the delamination segment, $C$ is a column vector of twelve arbitrary constants, and $M$ is a $12 \times 12$ matrix containing entries made up of elastic and geometrical properties. For a nontrivial solution the determinant of $M$ has to vanish to yield:

$$
\operatorname{det}\left(M\left(E_{2}, E_{3}, \rho_{2}, \rho_{3}, B, H_{2}, H_{3}, \xi_{1}, \xi_{2}, \omega_{i}\right)\right)=0,
$$

where det denotes the determinant of matrix $M$. In (2.4), elastic and geometrical properties are assumed to be known quantities arising from regular measurement, and thus $\lambda_{i}$ is simply and solely related to the delamination parameters, $\xi_{1}$ and $\xi_{2}$. This relation sets up a paradigm of delamination identification, briefed as follows. (1) Modal frequencies $\omega_{i}, i=1,2, \ldots, k$ are acquired by actual measurement, (2) $\omega_{i}, i=1,2, \ldots, k$, are individually inputted into (2.4) to establish a simultaneous system of characteristic equations, and (3) the simultaneous system is solved to produce the estimate of delamination parameters. In spite of clear technology route for the paradigm, the treatment of the simultaneous system is extremely complicated due to tremendous complexity of transcendental equation (2.4). Herein, to cope with the simultaneous system, an optimization strategy is adopted, of which the objective function is expressed as

$$
y\left(\xi_{1}, \xi_{2}\right)=\sum_{i=1}^{k} \log 10\left(\left|\operatorname{det}\left(M_{i}\left(\omega_{i}, \xi_{1}, \xi_{2}\right)\right)\right|+1\right)
$$

where $M_{i}$ is specified by measured $\omega_{i}$, in which the known quantities of elasticity and geometry are omitted for clarity. To estimate $\xi_{1}$ and $\xi_{2}$, a particular optimization problem is defined by

$$
\min _{\xi_{1}, \xi_{2}} y\left(\xi_{1}, \xi_{2}\right)=\min _{\xi_{1}, \xi_{2}}\left[\sum_{i=1}^{k} \log 10\left(\left|\operatorname{det}\left(M_{i}\left(\omega_{i}, \xi_{1}, \xi_{2}\right)\right)\right|+1\right)\right] \text { such that } 0<\xi_{1}<\xi_{2}<1
$$

Two modal frequencies, matching two unknowns $\xi_{1}$ and $\xi_{2}$, are ideally suited to set up the optimization problem as defined in (2.6). Considering the uncertainty in solving optimization problem, three or even more modal frequencies are needed to increase robustness in problem description, leading to an intricate optimization problem. 


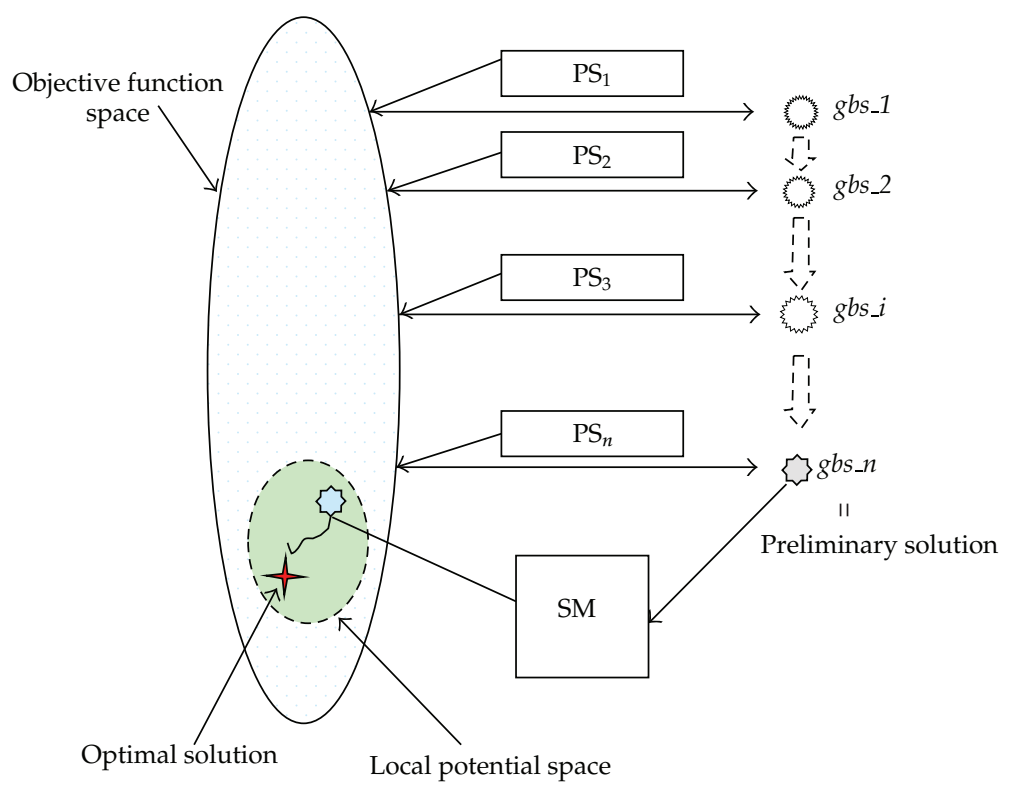

Figure 2: Illustrations of "global screening" and "local canvassing" of HOA. The dashed arrows symbolize evolution of interim global best solutions, filled polygon signifies preliminary solution, and star denotes optimal solution. (PS: particle swarm; SM: simplex method).

\section{Hybrid Optimization Algorithm}

A hybrid optimization algorithm (HOA) is proposed to solve the optimization problem for delamination identification. The HOA is established based on combining two well-known optimization methods: particle swarm optimization (PSO) [24-26] and simplex method (SM) [22]. The PSO has conspicuous capability in global search while the SM owns distinctive merit in local search $[22,24]$. As a combinative offspring, the HOA features a hierarchical and cooperative regime of global search using PSO and local search relying on SM. The global and local searches refer to "global screening" and "local canvassing," respectively. The embodiment of the HOA is presented in Figure 2, and the procedure to implement the algorithm is briefly described as follows.

\subsection{Global Screening}

A sequence of particle swarms (PSs) performs "global screening" for objective function space to achieve a preliminary solution. The intention of using sequential PSs rather than a single PS for global screening is to enhance the diversity of particles, ensuring a thorough global search. The main steps involved in the operation of global screening are briefed as the following.

(i) $\mathrm{PS}_{1}$ goes through the objective function space in a straight way, that is, with no iteration operations, to get the first interim global best solution, $g b s \_1$. "Interim" is used to distinguish from the final global best solution of optimization, that is, optimal solution. 
(ii) $\mathrm{PS}_{i}, 1<i<n$, examines the objective function space with possible iterations until it obtains an interim global best solution, gbs_i, satisfying that $g b s_{-} i$ just exceeds $g b s_{-}$ $(i-1)$.

(iii) Similar to that in (ii), $\mathrm{PS}_{n}$ brings forth $g b s \_n$, termed preliminary solution.

The sequence, $g b s_{\_} 1, g b s_{\_} 2, \ldots, g b s_{-} i, \ldots, g b s \_n$, manifests the evolution of interim global best solutions. In this process, the method of PSO fully exerts its advantage in global search to realize a preliminary solution by avoiding local minima in all probability.

\subsection{Local Canvassing}

The diversity and evolution of the global screening makes the preliminary solution potentially specify a local potential space probably bearing an optimal solution. The local potential space provides the SM a platform to explore optimal solution without the interference of dropping into local minima. The SM, launched by the preliminary solution, canvasses the local potential space to reach an optimal solution in a smooth and efficient manner.

As a hybrid algorithm, the HOA tactically consolidates the good character of PSO and that of SM while evading their respective deficiencies, forming an enhanced strategy for optimization identification of delamination in composite beams.

\subsection{Algorithm Verification}

The HOA is demonstrated on the delamination scenario VI in Table 1. As a preliminary work, the objective function involving first two modal frequencies is calculated as a forward analysis on sparse grid over domain $0<\xi_{1}<\xi_{2}<1$ as described in (2.6). The negative objective function is visualized in Figure 3, where the dominant peak signifying the global minimum is caused by $\xi_{1}=0.4$ and $\xi_{2}=0.6$, that is, the actual parameters of delamination. The striking protrusion of the peak in objective function space indicates the effectiveness of the objective function in reflecting delamination.

As an inverse analysis, the objective of the HOA is to optimize the objective function by adjusting variables $\xi_{1}$ and $\xi_{2}$ to determine delamination parameters, that is, to approach the peak in Figure 3. On the stage of global screening, $\mathrm{PS}_{1}, \mathrm{PS}_{2}, \ldots, \mathrm{PS}_{15}$, each comprising thirty particles, are employed to screen the objective function space as per the steps stated in Section 3.1. The actions of the $\mathrm{PS}_{1}, \mathrm{PS}_{3}$, and $\mathrm{PS}_{15}$ are exemplified in Figures 3, 4, and 5, respectively, in which three red balls mark the interim global best solutions, gbs_1, gbs_3, and $g b s_{-} 15$, respectively, and the black asterisks represent particles. $g b s_{-} 15$, with fitness function value -3.092, gives the preliminary solution. From Figure 3 to Figure 5, the interim global best solutions (red balls) gradually approach the optimal solution (striking peak), accounting for the evolution of interim global best solutions. On the stage of local canvassing, the SM initialized by the preliminary solution swiftly converges at an optimal solution, $\xi_{1}^{*}=0.4001$ and $\xi_{2}^{*}=0.6002$ by canvassing the local potential space for fifty iterations. The convergence process of the SM is depicted by the solid curve in Figure 6, in which the stable segment of the solid curve labels a fitness function value -0.005 for the optimal solution. The distinction of fitness function values between the preliminary solution and the optimal solution indicates that the precision improvement induced by the SM.

In addition, the behavior of PSO to pursue an optimal solution is also exhibited. The $\mathrm{PS}_{15}$ is arranged to proceed from the preliminary solution, gbs_15. After 300 iterations, the $\mathrm{PS}_{15}$ 


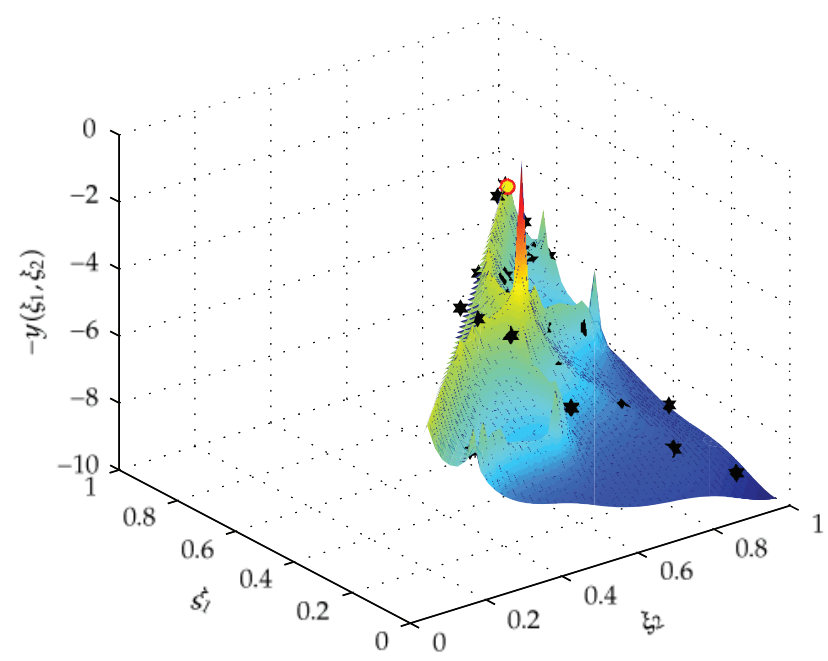

Figure 3: Interim global best solution, $g b s \_1$ (red ball), along with particle swarm (asterisks) distribution for $\mathrm{PS}_{1}$.

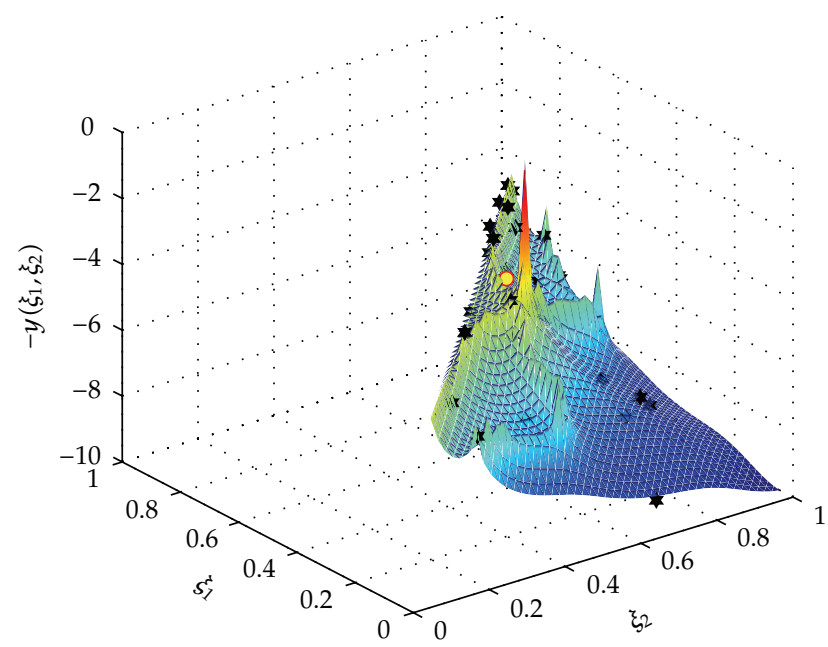

Figure 4: Interim global best solution, $g b s \_3$ (red ball), along with particle swarm (asterisks) distribution for $\mathrm{PS}_{3}$

converges at $g b s \_300$, with fitness function value being -0.01 , much cruder than -0.005 for the $\mathrm{SM}$. The convergence process is depicted by the dotted line in Figure 6. Clearly, the dotted curve is more zigzag than the solid one. The final particle swarm distribution of the $\mathrm{PS}_{15}$ in the $\left(\xi_{1}, \xi_{2}\right)$ plane is shown in Figure 7, in which the coordinates of $g b s \_300$ remarkably deviate from the point $\mathrm{A}$, the actual delamination parameters. These results imply the weaker capability of PSO in advancing the preliminary solution to an optimal solution.

The investigation above demonstrates the distinctive superiority of the HOA to individual PSO and SM. A module of software for implementing HOA has been developed in Matlab program language. 


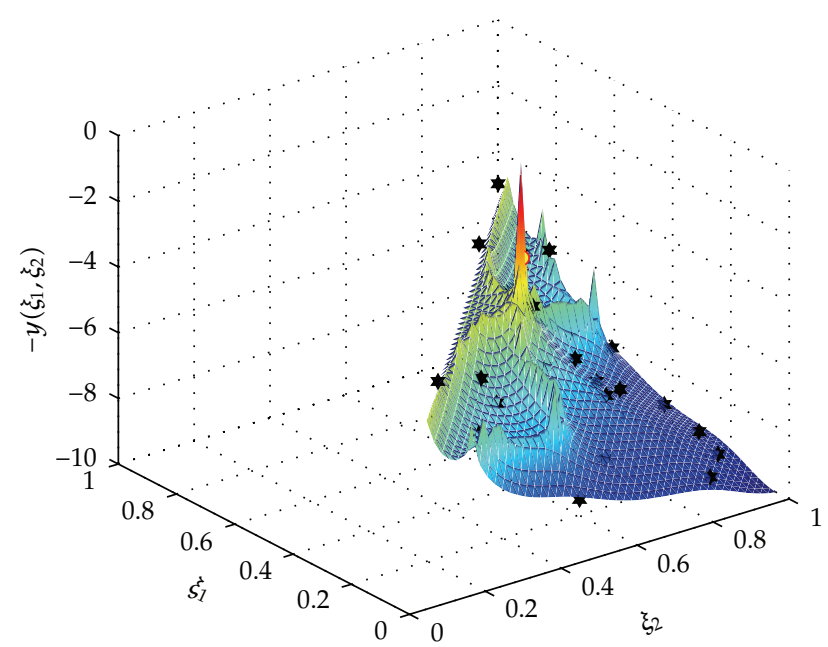

Figure 5: Preliminary solution, $g b s_{-} 15$ (red ball), along with particle swarm (asterisks) distribution for PS 15 .

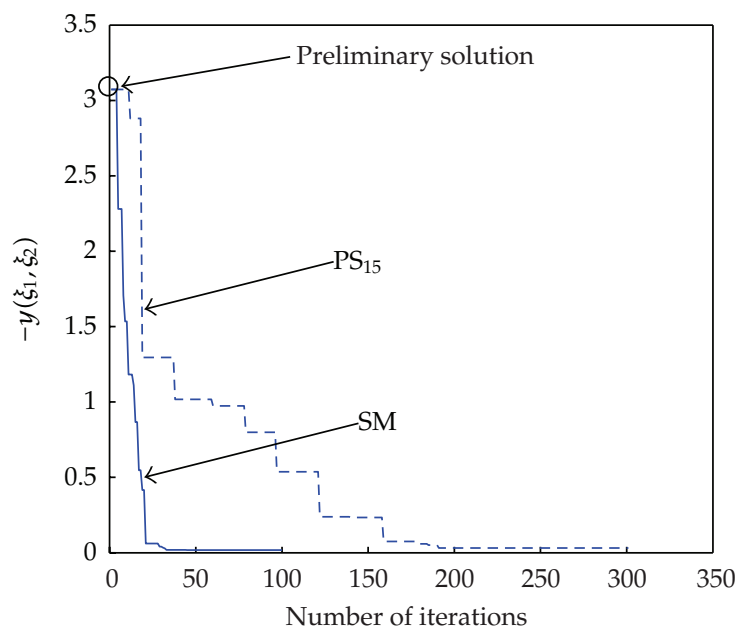

Figure 6: Convergence processes for SM (solid curve) and $\mathrm{PS}_{15}$ (dotted curve) starting with preliminary solution.

\section{Delamination Identification}

The proposed HOA is testified on a series of delamination scenarios (Table 1) concerning a double-layered clamped-clamped delaminated beam, shown in Figure 1. These scenarios are created by using the analytical mode of delaminated beam and changing the contrast ratio of densities, elastic moduli, or heights of Subbeams 2 and 3. The elastic and geometrical properties of the delaminated beam are given as, $L=21.175 \mathrm{~s}, H_{1}=0.18 \mathrm{~s}, B=0.25 \mathrm{in}, E_{3}=$ $31 \times 10^{6} \mathrm{psi}$, and $\rho_{3}=0.283 \mathrm{lb} / \mathrm{in}^{3}$, from which the other data can be generated in terms of the contrast ratio.

The first three modal frequencies, $\omega_{1}, \omega_{2}, \omega_{3}$, generated by analytical model using (2.1)-(2.4), with slight random noise, are used as experiment data acquired by a scanning laser vibrometer (SLV) [28] to set up an optimization problem (2.6). The resulting objective 


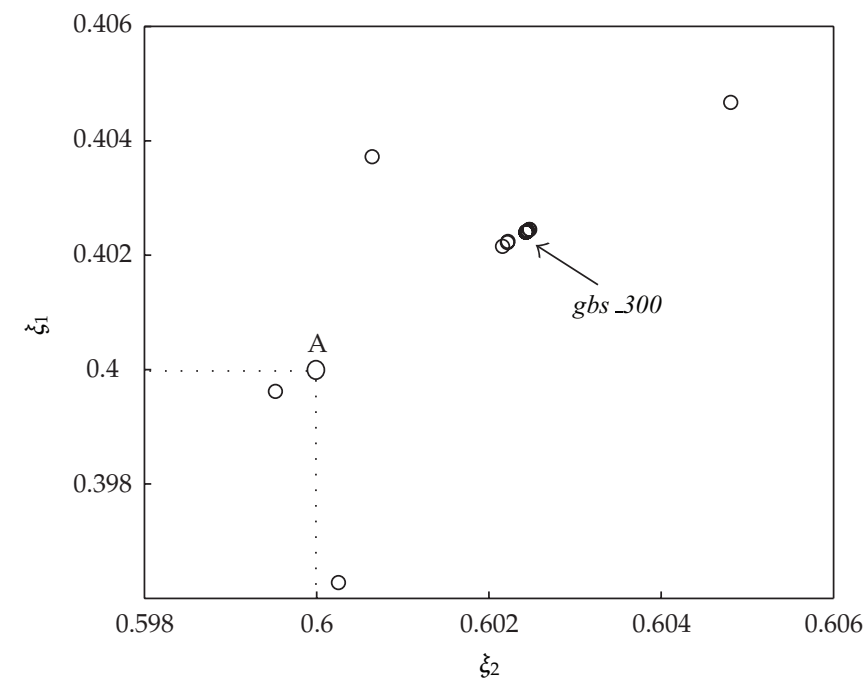

Figure 7: Particle swarm distribution in $\left(\xi_{1}, \xi_{2}\right)$ plane at the 300 th iteration for $\mathrm{PS}_{15}$ (Figure 6).

Table 1: Delamination scenarios and identification results from HOA.

\begin{tabular}{|c|c|c|c|c|}
\hline Case no. & $\xi_{1}, \xi_{2}$ & Delamination type & $\begin{array}{c}\omega_{1}, \omega_{2}, \omega_{3} \\
(\mathrm{~Hz})\end{array}$ & $\xi_{1}^{*}, \xi_{2}^{*}$ \\
\hline $\mathrm{I}$ & \multirow{5}{*}{$0.2,0.4$} & $E_{2}=E_{3}, \rho_{2}=\rho_{3}, H_{2}=H_{3}$ & $26.135,74.354,129.702$ & $0.200,0.400$ \\
\hline II & & $E_{2}=E_{3}, \rho_{2}=\rho_{3}, H_{2}=0.5 H_{3}$ & $26.454,74.504,135.005$ & $0.199,0.401$ \\
\hline III & & $E_{2}=E_{3}, \rho_{2}=\rho_{3}, H_{2}=0.2 H_{3}$ & $26.886,74.696,142.414$ & $0.200,0.401$ \\
\hline IV & & $E_{2}=0.5 E_{3}, \rho_{2}=\rho_{3}, H_{2}=H_{3}$ & $21.757,61.692,108.963$ & $0.201,0.400$ \\
\hline $\mathrm{V}$ & & $E_{2}=0.2 E_{3}, \rho_{2}=\rho_{3}, H_{2}=H_{3}$ & $15.980,44.757,82.814$ & $0.200,0.401$ \\
\hline VI & \multirow{5}{*}{$0.4,0.6$} & $E_{2}=E_{3}, \rho_{2}=\rho_{3}, H_{2}=H_{3}$ & $27.114,67.920,144.182$ & $0.400,0.600$ \\
\hline VII & & $E_{2}=E_{3}, \rho_{2}=\rho_{3}, H_{2}=0.5 H_{3}$ & $27.122,70.045,145.022$ & $0.399,0.599$ \\
\hline VIII & & $E_{2}=E_{3}, \rho_{2}=\rho_{3}, H_{2}=0.2 H_{3}$ & $27.131,73.024,146.074$ & $0.400,0.601$ \\
\hline IX & & $E_{2}=0.5 E_{3}, \rho_{2}=\rho_{3}, H_{2}=H_{3}$ & $22.484,56.889,119.783$ & $0.399,0.600$ \\
\hline$X$ & & $E_{2}=0.2 E_{3}, \rho_{2}=\rho_{3}, H_{2}=H_{3}$ & $16.278,42.751,87.291$ & $0.399,0.599$ \\
\hline
\end{tabular}

function is of considerable complexity, bringing difficulties for existing optimization methods. The HOA is utilized to tackle this optimization problem. As per the procedure described in Section 3, a sequence of fifteen PSs, each comprising thirty particles, are successively performed to get a preliminary solution. Afterwards, the SM, launched by this preliminary solution, is carried out to achieve an optimal solution. The identification results for a group of delamination cases are presented in Table 1. Comparing the estimated delamination parameters with the original ones, it can be concluded that the HOA has strong capability of identifying delamination in laminated composite beams.

\section{Conclusions}

A hybrid optimization algorithm (HOA) is proposed to deal with delamination identification in laminated composite structures. The HOA is based on combined particle swarm 
optimization (PSO) and simplex method (SM) and features several distinct characteristics: (1) the algorithm provides an innovative regime of hierarchical optimization by fusing typical global-search and local-search optimization methods; (2) the algorithm features consolidated merits of both PSO and SM while casting off their respective deficiencies; (3) the algorithm has strong adaptability for discrete and continuous optimization problems because of the property of free-of-derivative for PSO and SM; (4) the objective function of the HOA is underpinned by structural modal frequencies, allowing for low cost of measurement. The proposed method, aided by advanced sensing technology, holds promise for developing online systems of health monitoring for laminated composite beams.

\section{Acknowledgments}

M. Cao is grateful to a Marie Curie Industry Academia Partnership and Pathways Grant (Grant no. 251309 STA-DY-WI-CO) within the 7th European community Framework Programme, and acknowledges the partial support provided by a Foundation for the Author of National Excellent Doctoral Dissertation of PR China (Grant no. 201050) and a National Natural Science Foundation of China (Grant no. 50978084).

\section{References}

[1] P. M. Pawar and R. Ganguli, "Genetic fuzzy system for damage detection in beams and helicopter rotor blades," Computer Methods in Applied Mechanics and Engineering, vol. 192, no. 16-18, pp. 20312057, 2003.

[2] G. Z. Voyiadjis, Damage in Composite Materials, Elsevier, New York, NY, USA, 1993.

[3] Y. Zou, L. Tong, and G. P. Steven, "Vibration-based model-dependent damage (delamination) identification and health monitoring for composite structures-a review," Journal of Sound and Vibration, vol. 230, no. 2, pp. 357-378, 2000.

[4] P. Qiao, K. Lu, W. Lestari, and J. Wang, "Curvature mode shape-based damage detection in composite laminated plates," Composite Structures, vol. 80, no. 3, pp. 409-428, 2007.

[5] M. S. Cao and P. Z. Qiao, "Novel Laplacian scheme and multiresolution modal curvatures for structural damage identification," Mechanical Systems and Signal Processing, vol. 23, no. 4, pp. 1223-1242, 2009.

[6] Z. Wei, L. H. Yam, and L. Cheng, "Detection of internal delamination in multi-layer composites using wavelet packets combined with modal parameter analysis," Composite Structures, vol. 64, no. 3-4, pp. 377-387, 2004.

[7] N. Hu, T. Shimomukai, C. Yan, and H. Fukunaga, "Identification of delamination position in cross-ply laminated composite beams using S0 Lamb mode," Composites Science and Technology, vol. 68, no. 6, pp. 1548-1554, 2008.

[8] Y. J. Yan, L. Cheng, Z. Y. Wu, and L. H. Yam, “Development in vibration-based structural damage detection technique," Mechanical Systems and Signal Processing, vol. 21, no. 5, pp. 2198-2211, 2007.

[9] C. R. Farrar, S. W. Doebling, and D. A. Nix, "Vibration-based structural damage identification," Philosophical Transactions of the Royal Society A, vol. 359, no. 1778, pp. 131-149, 2001.

[10] Q. Wang and N. Wu, "Detecting the delamination location of beam with a wavelet transform: an experimental study," Smart Materials and Structures, vol. 20, no. 1, Article ID 012002, 7 pages, 2011.

[11] Z. Su and L. Ye, "Lamb wave-based quantitative identification of delamination in CF/EP composite structures using artificial neural algorithm," Composite Structures, vol. 66, no. 1-4, pp. 627-637, 2004.

[12] Y. J. Yan and L. H. Yam, "Detection of delamination damage in composite plates using energy spectrum of structural dynamic responses decomposed by wavelet analysis," Computers $\mathcal{E}$ Structures, vol. 82, no. 4-5, pp. 347-358, 2004.

[13] S. J. Wildy, A. G. Kotousov, B. S. Cazzolato, and J. D. Codrington, "New damage detection technique based on governing differential equations of continuum mechanics. Part I: out-of-plane loading," in Proceedings of the 6th Australasian Congress on Applied Mechanics (ACAM 6 '10), Perth, Australia, 2010. 
[14] J. Lee, R. T. Haftka, O. H. Griffin, L. T. Watson, and M. D. Sensmeier, “Detecting delaminations in a composite beam using anti-optimization," Structural and Multidisciplinary Optimization, vol. 8, no. 2-3, pp. 93-100, 1994.

[15] A. Nag, D. R. Mahapatra, and S. Gopalakrishnan, "Identification of delamination in composite beams using spectral estimation and genetic algorithm," Smart Materials and Structures, vol. 11, no. 6, pp. 899-908, 2002.

[16] A. Maranon, P. D. Ruiz, A. D. Nurse, J. M. Huntley, L. Rivera, and G. Zhou, "Identification of subsurface delaminations in composite laminates," Composites Science and Technology, vol. 67, no. 13, pp. 2817-2826, 2007.

[17] S. Dey and A. Karmakar, "Natural frequencies of delaminated composite rotating conical shells—a finite element approach," Finite Elements in Analysis and Design, vol. 56, pp. 41-51, 2012.

[18] P. Tan and L. Tong, "Identification of delamination in a composite beam using integrated piezoelectric sensor/actuator layer," Composite Structures, vol. 66, no. 1-4, pp. 391-398, 2004.

[19] R. Matsuzaki and A. Todoroki, "Wireless detection of internal delamination cracks in CFRP laminates using oscillating frequency changes," Composites Science and Technology, vol. 66, no. 3-4, pp. 407-416, 2006.

[20] G. M. L. Gladwell, "Inverse problems in vibration," Applied Mechanics Reviews, vol. 49, no. 10, pp. S25-S34, 1996.

[21] R. Fletcher, Practical Methods of Optimization, A Wiley-Interscience Publication, John Wiley \& Sons, New York, NY, USA, 2000.

[22] J. A. Nelder and R. Mead, "A simplex method for function minimization," The Computer Journal, vol. 7, pp. 308-320, 1965.

[23] C. T. Kelley, Iterative Methods for Optimization, SIAM, Philadelphia, Pa, USA, 1999.

[24] K. E. Parsopoulos and M. N. Vrahatis, "Recent approaches to global optimization problems through particle swarm optimization," Natural Computing, vol. 1, no. 2-3, pp. 235-306, 2002.

[25] M. Clerc and J. Kennedy, "The particle swarm-explosion, stability, and convergence in a multidimensional complex space," IEEE Transactions on Evolutionary Computation, vol. 6, no. 1, pp. 58-73, 2002.

[26] O. Begambre and J. E. Laier, "A hybrid Particle Swarm Optimization-Simplex algorithm (PSOS) for structural damage identification," Advances in Engineering Software, vol. 40, no. 9, pp. 883-891, 2009.

[27] S. Timoshenko, Vibration Problems in Engineering, John Wiley \& Sons, New York, NY, USA, 1974.

[28] R. B. Bai, M. S. Cao, Z. Q. Su, W. Ostachowicz, and H. Xu, "Fractal dimension analysis of higher-order mode shapes for damage identification of Beam structures," Mathematical Problems in Engineering, vol. 2012, Article ID 454568, 16 pages, 2012. 


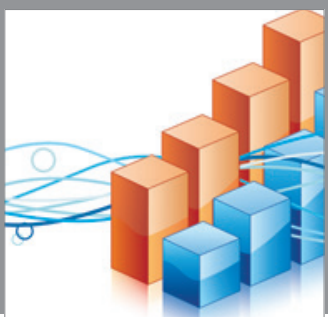

Advances in

Operations Research

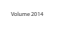

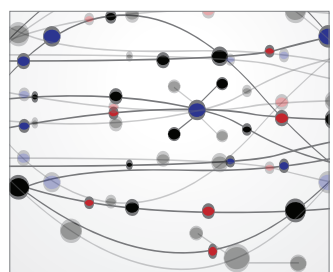

\section{The Scientific} World Journal
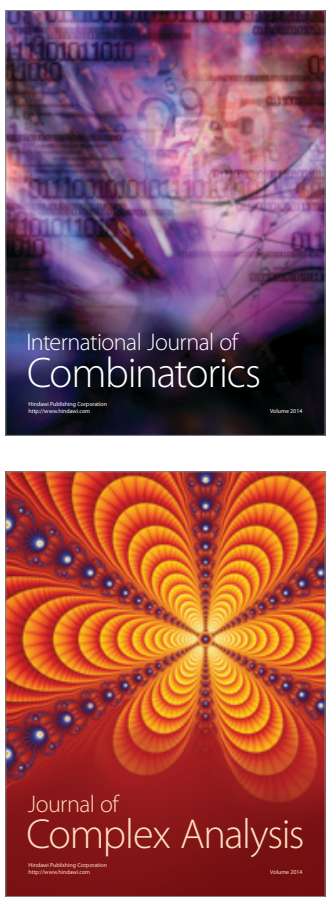

International Journal of

Mathematics and

Mathematical

Sciences
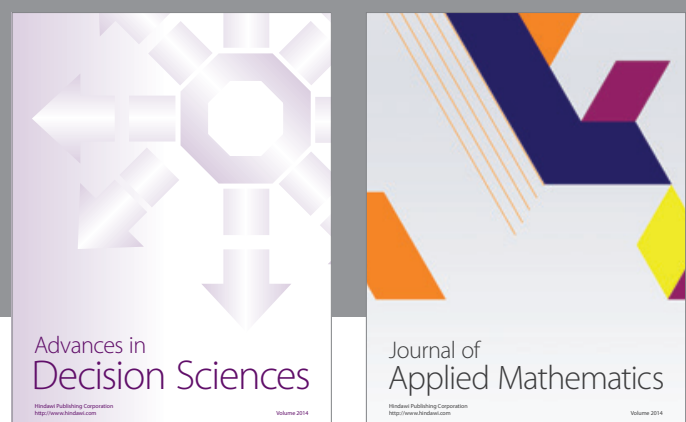

Journal of

Applied Mathematics
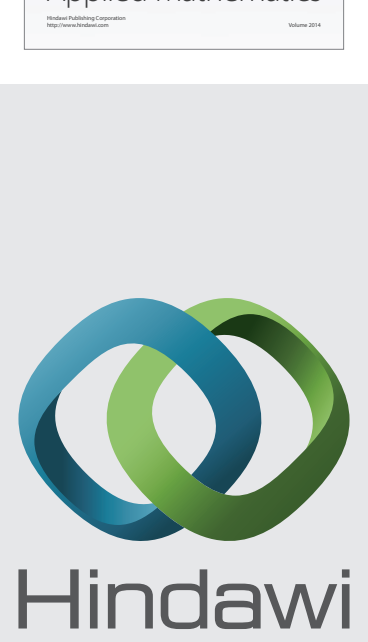

Submit your manuscripts at http://www.hindawi.com
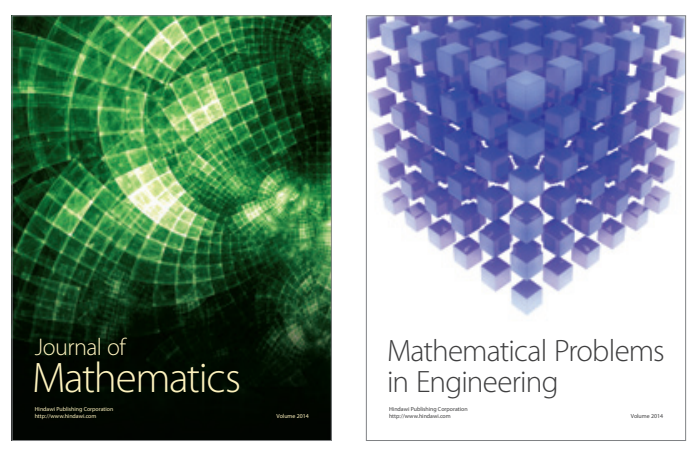

Mathematical Problems in Engineering
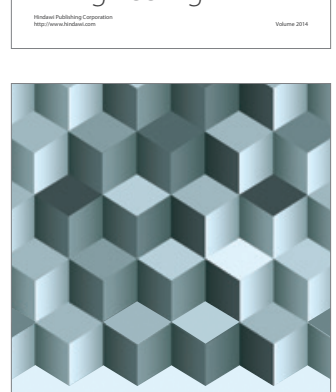

Journal of

Function Spaces
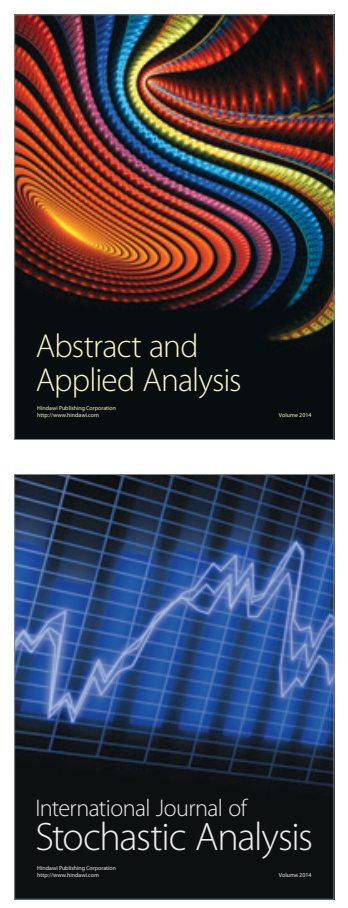

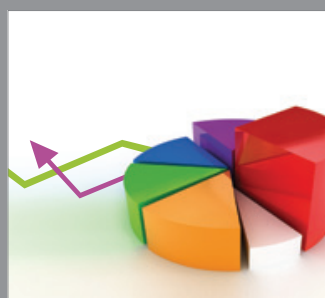

ournal of

Probability and Statistics

Promensencen
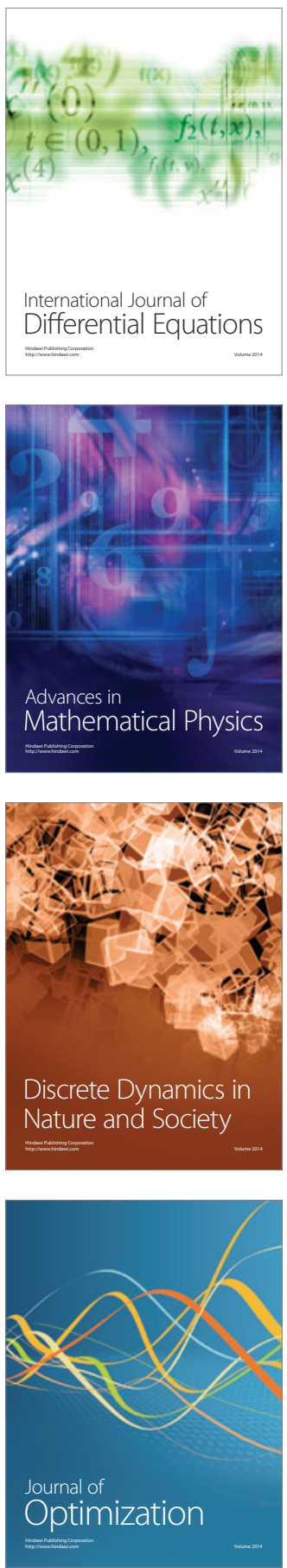